Check for updates

1 University of Fribourg, Fribourg Hospital, Fribourg, Switzerland

2 Geneva University Hospitals and Faculty of Medicine, Geneva, Switzerland

3 The University of Melbourne, Royal Children's Hospital Melbourne,

Parkville, Australia

Correspondence to: P Zimmermann petra.zimmermann@unifr.ch

Cite this as: BMJ 2022;376:0143 http://dx.doi.org/10.1136/bmj.0143 Published: 20 January 2022

\section{Long covid in children and adolescents}

\section{Risk appears low, but many questions remain}

\author{
Petra Zimmermann, ${ }^{1}$ Laure F Pittet, ${ }^{2}$ Nigel Curtis ${ }^{3}$
}

Symptoms involving almost every organ system have been reported after SARS-CoV-2 infection. ${ }^{1-3}$ Estimates of the prevalence of long covid (also called post-covid-19 condition, post-acute sequelae of covid-19, or chronic covid syndrome) vary considerably, partly because of confusion around the definition. The term long covid encompasses a broad range of symptoms, including objective complications of covid-19 (pulmonary fibrosis, myocardial dysfunction), mental health conditions, and more subjective, non-specific symptoms resembling those seen in post-viral chronic fatigue syndrome (myalgic encephalomyelitis). Most studies to date have substantial limitations, including small cohorts, absence of control groups, non-standardised capture of symptoms, lack of correction for pre-existing medical conditions, participant reported infection, and variation in follow-up, as well as selection, non-response, misclassification, and recall biases. ${ }^{4}$

In children and adolescents, acute covid-19 is less severe than in adults. ${ }^{56}$ Concern among many parents has therefore focused more on the potential long term effects of SARS-CoV-2 infection. Unfortunately, fewer data are available on long covid in young people compared with adults. ${ }^{7}$ The widely quoted one in seven frequency in children is based on a study with a $13 \%$ response rate. ${ }^{7} 8$

The linked study by Magnusson and colleagues (doi:10.1136/bmj-2021-066809) used nationwide register data from Norway to estimate the impact of covid-19 on long term healthcare use among 1.3 million children and adolescents. ${ }^{9}$ The authors identified a short term increase in primary (but not specialist) care use after covid-19 in all the studied age groups. This increase was related to respiratory and general or non-specific conditions, mostly in the four weeks after infection. The increase in primary care use persisted for up to six months among children aged 1-5 years. Notably, covid-19 in children had limited overall impact on healthcare services.

The study's strengths include its population based design, the inclusion of SARS-CoV-2 negative and non-tested control groups, and comparison with pre-pandemic healthcare use. An unavoidable limitation is that asymptomatic children or those with mild symptoms might not have been tested. Also, changes in testing patterns could have occurred in different age groups and over time, and children testing positive for SARS-CoV-2 might also have had greater exposure to other respiratory viruses. Finally, the anxiety surrounding this previously unknown infection in children might have caused primary care providers and parents to schedule unnecessary follow-up visits after a positive test result.
Magnusson and colleagues' study highlights the difficulty of accurately determining risk of long covid in children and adolescents, and the urgent need for further rigorous studies. Reports suggest that more than half of children who did not have covid-19 experienced symptoms such as headaches, fatigue, sleep disturbance, and concentration difficulties during the pandemic. ${ }^{7}$ Distinguishing long term symptoms caused by SARS-CoV-2 infection from pandemic related symptoms remains a challenge. One large study in the UK found that nearly all symptoms reported by children who tested positive for SARS-CoV-2 were also reported by those who tested negative. ${ }^{7}$ Moreover, no difference was reported between the two groups in mental health, overall wellbeing, or impairment of activities. Other studies with control groups have also reported minimal differences in persisting symptoms between children with SARS-CoV-2 infection and those without. ${ }^{4}$ This underlines the importance of appropriate control groups, including children with other infections and those admitted to hospital for other reasons.

In addition to the uncertainty around the true prevalence of long covid, several other important questions remain unanswered. Firstly, what are the risk factors for developing long covid? Although some studies in adults have suggested that severity of the initial infection, hospital admission, female sex, white ethnicity, middle age, and asthma are risk factors for persistent symptoms, ${ }^{121011}$ the most recent and comprehensive meta-analysis concluded that data were insufficient to determine the influence of these factors. ${ }^{3}$ Secondly, what are the molecular, immunological, and psychological mechanisms underlying long covid? Suggested mechanisms include direct effects of the virus (including viral latency, persistent activation of the immune system, ${ }^{12}$ and neuronal apoptosis) and indirect effects related to mental health problems such as post-traumatic stress and social isolation. ${ }^{13}$ Thirdly, are the long term effects of covid-19 specific to SARS-CoV-2 infection or are they similar to post-viral syndromes seen after other viral infections? Fourthly, can long covid be prevented? Recent studies in adults suggest that covid-19 vaccination is associated with a lower risk of several, but not all, sequelae in those with breakthrough SARS-CoV-2 infections. ${ }^{14} 15$ Finally, what is the optimal treatment? Even if risk of long covid is low, the high incidence of SARS-CoV-2 infections, especially with the omicron variant, means that large numbers of children might require treatment.

Currently a third of all children and adolescents reports negative emotions, such as sadness or anxiety, highlighting the toll from the pandemic in 
this age group. ${ }^{7}$ Vaccinating young people might help reduce some of the indirect harms caused by repeat testing and isolation, lockdowns, school closures, and reduced social activities. As SARS-CoV-2 remains predominantly a mild infection in the paediatric population, the incidence of long covid is a critical factor in the risk-benefit equation for policy and parental decisions on covid-19 vaccines for children. ${ }^{16}$

Competing interests: The BMJ has judged that there are no disqualifying financial ties to commercial companies. The authors declare the following other interests: None. Further details of The BMJ policy on financial interests is here: https://www.bmj.com/sites/default/files/attachments/re-

sources/2016/03/16-current-bmj-education-coi-form.pdf."

Provenance and peer review: Commissioned; not externally peer reviewed.

1 Iqbal FM, Lam K, Sounderajah V, Clarke JM, Ashrafian H, Darzi A. Characteristics and predictors of acute and chronic post-COVID syndrome: A systematic review and meta-analysis. EClinicalMedicine 2021;36:100899. doi: 10.1016/j.eclinm.2021.100899 pmid: 34036253

2 Lopez-Leon S, Wegman-Ostrosky T, Perelman C, etal. More than 50 long-term effects of COVID-19: a systematic review and meta-analysis. Sci Rep 2021;11:16144 doi: 10.1038/s41598-021-95565-8 pmid: 34373540

3 Groff D, Sun A, Ssentongo AE, etal. Short-term and Long-term Rates of Postacute Sequelae of SARS-CoV-2 Infection: A Systematic Review. JAMA Netw Open 2021;4:e2128568. doi: 10.1001/jamanetworkopen.2021.28568 pmid: 34643720

4 Zimmermann P, Pittet LF, Curtis N. How Common is Long COVID in Children and Adolescents? Pediatr Infect Dis / 2021:40:e482-7.

doi: 10.1097/INF.0000000000003328 pmid: 34870392

5 Zimmermann P, Curtis N. Coronavirus Infections in Children Including COVID-19: An Overview of the Epidemiology, Clinical Features, Diagnosis, Treatment and Prevention Options in Children. Pediatr Infect Dis /2020;39:355-68. doi: 10.1097/INF.0000000000002660 pmid: 32310621

6 Zimmermann P, Curtis N. Why is COVID-19 less severe in children? A review of the proposed mechanisms underlying the age-related difference in severity of SARS-CoV-2 infections. Arch Dis Child 2020;archdischild-2020-320338. pmid: 33262177

7 Stephenson T, Pereira SP, Shafran R, etal. Long COVID - the physical and mental health of children and non-hospitalised young people 3 months after SARS-CoV-2 infection; a national matched cohort study (The CLoCk) Study. Nature Portfolio 2021; doi: 10.21203/rs.3.rs-798316/v1

8 Wise J. Long covid: One in seven children may still have symptoms 15 weeks after infection, data show. BM/2021;374:n2157. doi: 10.1136/bmj.n2157 pmid: 34470745

9 Magnusson K, Skyrud KD, Suren P, etal. Healthcare use in 700000 children and adolescents for six months after covid-19: before and after register based cohort study. BMJ 2022;376:e666809. doi: 10.1136/bmj-2021-066809 pmid: 35039315

10 Munblit D, Bobkova P, Spiridonova E, etalSechenov StopCOVID Research Team. Incidence and risk factors for persistent symptoms in adults previously hospitalized for COVID-19. Clin Exp Allergy 2021;51:1107-20. doi: 10.1111/cea.13997 pmid: 34351016

11 Sigfrid L, Drake TM, Pauley E, etallSARIC4C investigators. Long Covid in adults discharged from UK hospitals after Covid-19: A prospective, multicentre cohort study using the ISARIC WHO Clinical Characterisation Protocol. Lancet Reg Health Eur 2021;8:100186. doi: 10.1016/j.lanepe.2021.100186 pmid: 34386785

12 Phetsouphanh C, Darley DR, Wilson DB, etal. Immunological dysfunction persists for 8 months following initial mild-to-moderate SARS-CoV-2 infection. Nat Immunol 2022; published online 13 January. doi: 10.1038/s41590-021-01113-x pmid: 35027728

13 Groff D, Sun A, Ssentongo AE, etal. Short-term and Long-term Rates of Postacute Sequelae of SARS-CoV-2 Infection: A Systematic Review. JAMA Netw Open 2021;4:e2128568. doi: 10.1001/jamanetworkopen.2021.28568 pmid: 34643720

14 Taquet M, Dercon Q, Harrison PJ. Six-month sequelae of post-vaccination SARS-CoV-2 infection: a retrospective cohort study of 10,024 breakthrough infections.medRxiv 2021:2021.10.26.21265508doi: 10.1101/2021.10.26.21265508.

15 Antonelli M, Penfold RS, Merino J, etal. Risk factors and disease profile of post-vaccination SARS-CoV-2 infection in UK users of the COVID Symptom Study app: a prospective, community-based, nested, case-control study. Lancet Infect Dis 2022;22:43-55. doi: 10.1016/S1473-3099(21)00460-6 pmid: 34480857

16 Zimmermann P, Pittet LF, Finn A, Pollard AJ, Curtis N. Should children be vaccinated against COVID-19?. Arch Dis Child 2021; published online 3 November. doi: 10.1136/archdischild-2021-323040 pmid: 34732388

This article is made freely available for personal use in accordance with BMJ's website terms and conditions for the duration of the covid-19 pandemic or until otherwise determined by BMJ. You may download and print the article for any lawful, non-commercial purpose (including text and data mining) provided that all copyright notices and trade marks are retained. 